\title{
Slant judgments of single rectangles at a slant'
}

\author{
HOWARD R. FLOCK, YORK UNIVERSITY; DAVID GRAVES, DARTMOUTH COLLEGE; \\ JAMES TENNEY, HARVARD UNIVERSITY; BRUCE STEPHENSON, YORK UNIVERSITY
}

Twelve rectangles varying in angular height from $54^{\circ}$ to $1.2^{\circ}$ were presented singly at 4 to 6 slants. Accuracy of slant judgments decreased with angular height and reached chance level with the $1.2^{\circ}$ shape. The results are contrasted to the output of other laboratories.

Over the past 10 years particularly, and earlier, a body of literature has developed giving the relation between slanted solitary rectangles of varying sizes and shapes and judgments of their slant. (See Flock, 1965 for a review of some of these experiments.) When one compares the experimental outputs from different laboratories the past evidence seems to indicate that as a rectangle becomes angularly smaller, judgments of its slant become better rather than worse (see Table 1, Flock, 1965). Thus, one finds that as textureless solitary rectangles go from $10^{\circ}$ in angular height to 45 minutes of arc, the accuracy of slant judgments increases from being about $35 \%$ correct to being about $100 \%$ correct. This state of affairs implies, at least for rectangles, that as they become smaller and approach invisibility, judgments of their slant become better and better. The main objective of this paper, therefore, is to determine what happens to judgmental accuracy as rectangles that are slanted are made smaller and smaller. A related objective is to determine whether there are any conditions under which individual rectangles that are slanted elicit approximately accurate judgments of their slant.

\section{Stimulus displays and apparafus}

Twelve planar rectangles were cut from heavyduty vinyl contact paper. Six of the rectangles (Groups $I$ and II) had a height-base (H/B) ratio of $1.33: 1$ and six (Groups III and IV) a $\mathrm{H} / \mathrm{B}$ ratio of $2.5: 1$. The areas in square inches of the six rectangles in Groups I and $I I$ were $36.8,5.7,1.3, .9, .4$, and .06; and of the six rectangles in Groups III and IV, 26.4, 3, .8, .2, .05 , and .01 . The rectangles were varied, therefore, from relatively large to quite small areas.

Each rectangle was mounted on a transparent sheet of Plexiglas so that when viewed in a frontal position by $\mathrm{a} \mathrm{S}$ it would be exactly in the middle of his field of view. The sheets of Plexiglas were placed 8 in. in front of a $25-\mathrm{w}$ Sylvania zirconium point-source, at a distance of 27.5 in. from a $7 \times 7 \mathrm{ft}$. translucent Plexiglas projection-screen. By shifting the mount in which the Plexiglas sheets were placed each rectangle could be located so that it was normal to the light and parallel to the screen. When the six rectangles in Groups I and II were in a frontal-parallel position their angular sizes in the vertical meridian were $47^{\circ}, 20^{\circ}, 10^{\circ}, 8^{\circ}, 5^{\circ}$, and $2^{\circ}$; the corresponding angular sizes for the six rectangles in Groups III and IV were $54^{\circ}, 20^{\circ}, 10^{\circ}, 5^{\circ}, 2.5^{\circ}$, and $1.2^{\circ}$. With that range and variety, the sample of rectangles was more representative of the population of rectangles than had been the case heretofore (Flock, 1965).

The total angular view of the shapes was controlled by diaphragms in front of the point-source and in front of the viewing position. (See Table 1 below for the angular views.)

The Plexiglas sheets could be placed at any one of several slants from the frontal-parallel position so as to produce a floor slant (as when a surface is slanted with its top away from one) or a ceiling slant (as when a surface is slanted with its top towards one). The projected shapes, at a distance of 35.5 in. from the $\mathrm{S}$, were monocularly viewed through a special eye-piece that minimized parallactic head movements. This projection method eliminated binocular cues and controlled for accommodation. (See Flock, 196 tb, for a more detailed description of the method.)

\section{Procedure}

Twenty-four male undergraduates at Dartmouth College and it undergraduates at York University, all with $20 / 20$ uncorrected vision in at least one eye, were subdivided into four equal groups. Each group was assigned to a set of three shapes: Groups I and II (at Dartmouth) to the three largest and three smallest 1.33:1 rectangles, and Groups III and IV (at York) to the three largest and three smallest 2.5:1 rectangles, respectively. At Dartmouth each shape was presented at two floor $\left(30^{\circ}\right.$ and $\left.15^{\circ}\right)$ and two ceiling $\left(30^{\circ}\right.$ and $\left.15^{\circ}\right)$ slants. At York each shape was presented at three floor $\left(30^{\circ}, 20^{\circ}\right.$, and $\left.10^{\circ}\right)$ and three ceiling $\left(30^{\circ}, 20^{\circ}\right.$, and $10^{\circ}$ ) slants. Each Dartmouth $\mathrm{S}$ made 12 judgments and each York S, 18 judgments. (The York experiments were designed to test the Dartmouth results with a different set of rectangles, with more slants, and with different experimenters; and were run a year later.) The order of presentation of the three shapes was counter-balanced over each group, whereas the order of presentation of the sequence of slants was randomized.

Each $\mathrm{S}$ was required to examine a shape when slanted and "from your impression of its slant" was required to rotate a diameter to that angle of rotation which exactly matched the angle of slant of the shape. Ss were specifically told to make their judgments on the basis of the appearance of the slant of the shape and were told to avoid trying to figure out the slant of the shape. In making this judgment $S$ had to turn his head $90^{\circ}$ and rotate in a clockwise or counterclockwise direction an 8-in. diameter that was normal to his 
Table 1. Parameters of 12 rectangles and slant judgments expressed as median $b \mathbf{S}, Q \mathbf{s}$, and $\tau \mathbf{S}$

\begin{tabular}{|c|c|c|c|c|c|c|c|c|c|c|}
\hline \multirow[t]{2}{*}{ Group } & \multirow{2}{*}{$\begin{array}{l}\text { Area } \\
\text { (in) }\end{array}$} & \multicolumn{2}{|c|}{$H T$ in $(9)$} & \multirow{2}{*}{$\begin{array}{c}\text { View } \\
19 \\
\end{array}$} & \multicolumn{2}{|c|}{ Median $b$} & \multicolumn{2}{|c|}{$Q$} & \multicolumn{2}{|c|}{ Median $r \& Q$} \\
\hline & & $Y$ & & & $Y$ & & $Y$ & $D$ & & \\
\hline III & 26.4 & 54 & & 71 & .69 & & .15 & & $.87 / .06$ & \\
\hline 1 & 36.8 & & 47 & 67 & & .71 & & .16 & & $1.0 / 0$ \\
\hline III & 3. & 20 & & 24 & .48 & & .12 & & $.92 / .10$ & \\
\hline I & 5.7 & & 20 & 40 & & .67 & & .22 & & $1.0 / 16$ \\
\hline 111 & .76 & 10 & & 14 & .20 & & .14 & & $.76 / .24$ & \\
\hline 1 & 1.33 & & 10 & 21 & & .29 & & .08 & & $1.0 / .16$ \\
\hline II & .94 & & 8 & 18 & & .34 & & .14 & & $1.0 / .02$ \\
\hline IV & .19 & 5 & & 8 & .10 & & .14 & & $.62 / .38$ & \\
\hline II & .36 & & 5 & 9 & & .21 & & .08 & & $.79 / .16$ \\
\hline IV & .05 & 2.5 & & 7 & .09 & & .07 & & $.07 / .14$ & \\
\hline II & .06 & & 2 & 8 & & .13 & & .09 & & $.33 / .29$ \\
\hline IV & .01 & 1.2 & & 2 & -.06 & & .23 & & $0 / .48$ & \\
\hline
\end{tabular}

visual axis and that subdivided an aluminum disc into black and white halves.

A S's sequence of slant judgments over the four or six slants of a shape was described by a linear regression coefficient, b. For each $\mathrm{S}$ there were three regression coefficients, one for each of the three shapes to which he was exposed. A median regression coefficient was then calculated for each shape. A coefficient of 1.0 was taken as the criterion of a set of accurate judgments of slant. Departures from 1.0 in any direction were taken to be error. As a rough measure of the goodness of fit of a straight line to the slant judgments, Kendall's Tau (Siegel, 1956) was calculated for each S's sequence of judgments over the slants of a shape. A Tau ( $T$ ) of 1.0 was taken to mean that a S's data were approximately linear and perfectly monotonic. A $\tau$ of 0 , correspondingly, indicated that the slant judgments were at chance level. Semi-interquartile ranges (Q) were calculated for the bs and $\tau s$.

\section{Results and Discussion}

The top half of Table 1 describes the results from York (Y) and Dartmouth (D) for the large rectangles; the bottom half describes the results for the small rectangles. ${ }^{2}$ Scanning down the column of median bs for York, one will note that as the angular height of a rectangle decreases so does the median $b$, reaching the chance level of 0 when angular height was $1.2^{\circ}$. This same trend, except for a reversal between the $8^{\circ}$ and $10^{\circ}$ rectangles, occurred also at Dartmouth (and is reflected in the $T^{\prime} s^{3}$ ).

These trends in the median bs seem to imply that as angular height of rectangles is decreased, judgments of their slant decrease towards a chance level. It would seem, therefore, that many of the results from the laboratories cited earlier were probably specific to the methods under which they were observed.

Why were the slant judgments not better than they were? No median regression coefficient was greater than .71 , and only 10 of the 144 individual regression coefficients were greater than .83 . (The two largest rectangles contributed seven of these). Theoretically, three angular widths in the projected trapezoid are sufficient for correct slant perceptions: the angular widths, $\phi, \gamma$, and $\alpha$, of the shorter trapezoidal base, of the trapezoid at the point corresponding to the objective center of the rectangle when slanted on its horizontal axis, and of the trapezoid at that place where the locations of $\phi$ and $\alpha$ are angularly equidistant from $Y$, respectively. Since the projection of the objective center of a slanted rectangle can not be optically identified in a projected trapezoid, one can substitute the center of the trapezoid for the objective center of the slanted rectangle. This substitution should not theoretically produce an error in excess of that indicated by a regression coefficient of .82 for any of the rectangles at any of the slants used in the above or in earlier studies. (See Flock, 1965, note 6 and Flock, 1964a, pp. 387-389, for the method that will derive these expectations.) Neither in the study above nor in earlier studies does one tend to find regression coefficients as large as .82 (except for possible artifacts as indicated in Flock, 1965). One might conclude from this that the single untextured rectangular shape when viewed monocularly without parallax constitutes too great a degree of impoverishment to elicit accurate slant judgments from the human visual system, regardless of its angular size. (See Flock, $1964 a, 1965$, for a discussion of sufficient conditions for eliciting accurate slant judgments.)

\section{References}

Flock, H. R. A possible optical basis for monocular slant perception. Psychol. Rev., 1964a, 71, 380-391.

Flock, H. R. Some conditions sufficient for accurate monocular perceptions of moving surface slants. J. exp. Psychol., 1964b, 67, 560-572.

Flock, H. R. Optical texture and linear perspective as stimuli for slant perception. Psychol. Rev., 1965, 72, 505-514.

Siegel, S. Nonparametric methods for the behavioral sciences. New York: McGraw-Hill, 1956

\section{Notes}

1. This research was supported by the National Science Foundation Research Grant No. GB 2474 and by the National Research Council of Canada Grant No. APA-143.

2. The tables of raw data from which these statistics were calculated may be obtained at no charge from the Editorial office of this Journal.

3. The higher $r$ s at Dartmouth probably reflect the greater separation between successive slants. 